\title{
In situ ETEM study of surface reconstruction formation on stepped Cu surfaces during oxidation
}

\author{
Meng $\mathrm{Li}^{1}$, Matthew Curnan ${ }^{2}$, Richard Garza ${ }^{3}$, Stephen House ${ }^{1}$, Wissam Saidi ${ }^{3}$ and Judith Yang ${ }^{3}$ \\ ${ }^{1}$ University of Pittsburgh, Pittsburgh, Pennsylvania, United States, ${ }^{2}$ University of Pittsburgh, \\ PITTSBURGH, Pennsylvania, United States, ${ }^{3}$ University of Pittsburgh, United States
}

Surface reconstruction is the process through which the crystal structure of surface atoms changes from bulk to other structures, when there are absorbates such as gas molecules on the surface. This is an important step in surface chemistry that is critical for applications in catalysis and corrosion. During metal oxidation, surface reconstruction is commonly observed in many metals and alloys, and is considered a key step for the ensuing oxide nucleation and growth process. However, the role of surface reconstruction on oxidation remains debated due to a lack of experimental verification. Surface reconstruction is usually studied using Scanning Tunneling Microscopy. Limited by the time resolution of this technique, the dynamic processes of surface reconstruction formation and evolution remain unclear. On realistic surfaces, defects such as surface steps are omnipresent and have been found to affect interfacial atom diffusion and absorbate distribution. This could impact reconstruction or even later oxidation processes.[1,2] Although multiple surface reconstructions during oxidation have been reported, the dynamic processes forming surface reconstructions, and the effects of surface defects such as surface steps on the reconstruction process, are still unclear.

In this work, we show that in situ Environmental TEM could be a powerful tool to investigate surface reconstruction dynamics during oxidation. Using atomic resolution in situ Environmental TEM with precisely controlled low $\mathrm{O} 2$ pressure, the dynamic formation process of surface reconstruction on stepped $\mathrm{Cu}$ surfaces is investigated. In situ ETEM observation was performed using a Hitachi H-9500 ETEM, with a Hitachi double-tilt heating holder and a homemade gas delivery system with three gas injection lines. The gas injection lines are connected to the pumping system of the ETEM, so that the residual gas in the pipeline can be quickly removed and gas exchange can be finished within minutes. Single crystalline $\mathrm{Cu}(100)$ thin films with nominal thicknesses of $60 \mathrm{~nm}$ were annealed in $\mathrm{H} 2$ in the ETEM to create oxidefree pure $\mathrm{Cu}$ surfaces with faceted holes. These faceted holes enable observation of the surface reconstruction dynamics from the cross-section view, as well as creating many surface steps to observe their effects on the reconstruction dynamics. After forming these faceted holes, samples were cooled to $300{ }^{\circ} \mathrm{C}$ and $\mathrm{O} 2$ was meticulously injected with very low pressure to observe the initial surface reconstruction formation process. Reconstruction formation on stepped $\mathrm{Cu}(100)$ and (110) facets are comparatively studied. As shown in Figure 1(a-c), Missing Row Reconstruction (MRR) on $\mathrm{Cu}(100)$ surfaces is observed to preferably form on the upper terraces of surface steps, followed by formation on the lower terraces. The flat $\mathrm{Cu}(100)$ surface is the last place to form MRR. In contrast, for $\mathrm{Cu}(110)$ surfaces, as shown in Figure 1(d-f), ( $2 \times 1)$ reconstructions are observed on both upper and lower terrace sites without obvious preference. Our presentation will discuss the oxidation preferences, mechanisms, and dynamics undergone by observed surface reconstruction formation processes, further developing the experimental work depicted in Figure 1. Correlated computational efforts will provide atomistic insights into these preferences and mechanisms, namely by applying density functional theory, nudged elastic band, and related methods. Such results would enhance understanding of surface reconstructions and the ensuing oxidation process, providing a powerful and promising way to study dynamics during surface reconstruction. 

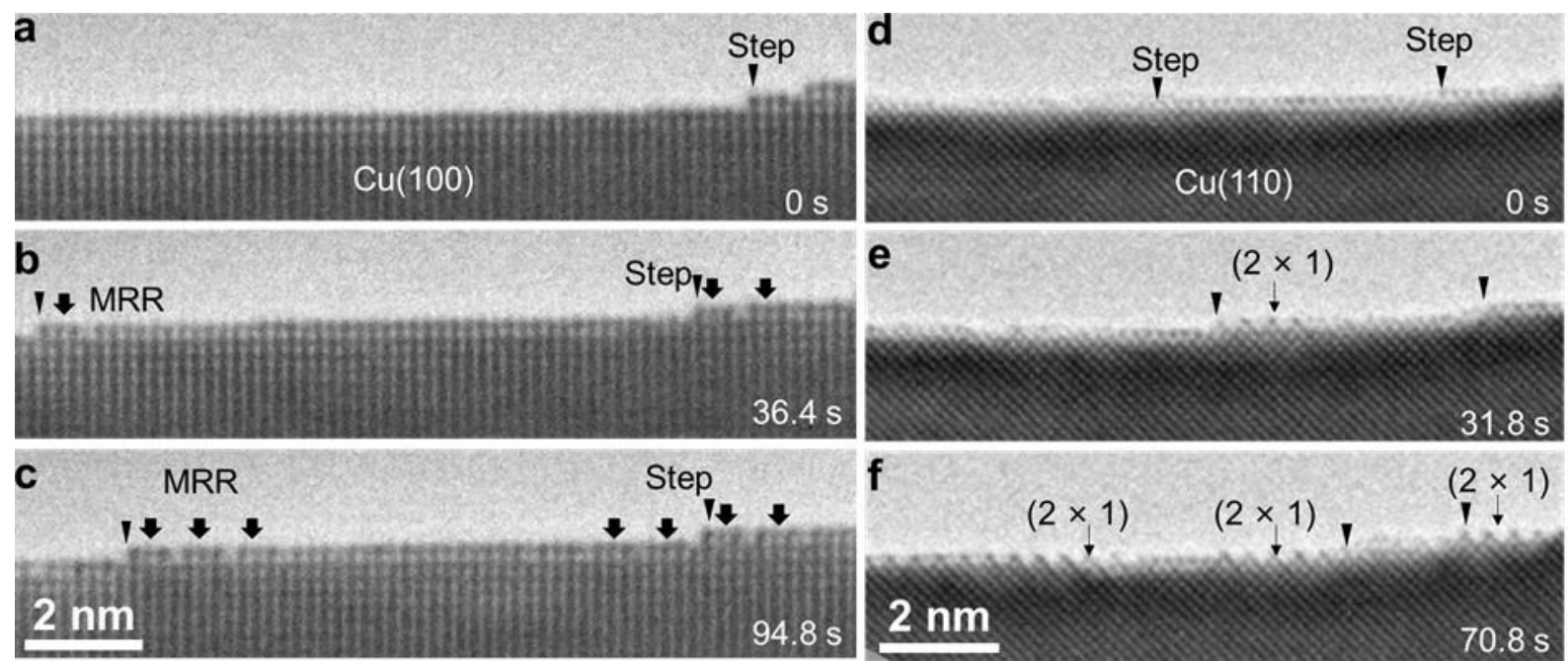

Figure 1. In situ ETEM observation of surface reconstruction formation on stepped Cu surfaces. (a-c) HRETEM observation of the formation of Missing Row Reconstruction (MRR) on stepped Cu(100) surface. (d-f) HRTEM observation of the formation of $(2 \times 1)$ reconstruction on stepped $\mathrm{Cu}(110)$ surface. The surface steps and reconstructions are marked by triangles and arrows, respectively.

\section{References}

[1] Q. Zhu, W. A. Saidi, J. C. Yang, Enhanced Mass Transfer in the Step Edge Induced Oxidation on Cu(100) Surface. J. Phys. Chem. C. 121, 11251-11260 (2017).

[2] M. T. Curnan, C. M. Andolina, M. Li, Q. Zhu, H. Chi, W. A. Saidi, J. C. Yang, Connecting Oxide Nucleation and Growth to Oxygen Diffusion Energetics on Stepped Cu(011) Surfaces: An Experimental and Theoretical Study. J. Phys. Chem. C. 123, 452-463 (2019).

[3] The authors acknowledge funding from National Science Foundation (NSF) grants DMR-1410055, DMR-1508417, DMR-1410335, and CMMI-1905647, as well as support from Hitachi-High-Tech and technical assistance from the Nanoscale Fabrication and Characterization Facility (NFCF) in the Petersen Institute of Nano Science and Engineering (PINSE) at the University of Pittsburgh. 